\title{
DIAGNOSTICS OF HELIUM-ARGON ARC DISCHARGE PLASMA BASED ON SPECTRAL LINE SHAPE MEASUREMENTS
}

\author{
T. WUJEC AND J. MUSIELOK \\ Institute of Physics, Opole University, Oleska 48, 45-052 Opole, Poland
}

(Received October 2, 1998; in final form November 27, 1998)

\begin{abstract}
Electron densities and ion (gas) temperatures on the axis of an arc discharge plasma, produced at atmospheric pressure in a gas mixture of $95 \%$ helium and $5 \%$ argon, are determined at two arc currents. The evaluation of both main plasma parameters is based on line shape measurements, the ion temperature on the Doppler broadening of selected ArII lines, while the electron density on the Stark broadening of the hydrogen $\mathrm{H}_{\beta}$ line which appear in the spectrum due to hydrogen traces in the applied gases. The significance of reliable plasma diagnostics for determination of atomic structure data is discussed.
\end{abstract}

PACS numbers: $32.30 .-\mathrm{r}, 32.70 .-\mathrm{n}, 52.25 .-\mathrm{b}$

\section{Introduction}

Helium arc discharges have been recently often applied as excitation sources for various elements introduced into the plasma as very small admixtures. Measurements of intensities of spectral lines of these species allow one, e.g., to determine relative transition probabilities for these lines [1-4]. Usually the relative $A_{k i}$-values or branching ratios are then normalized to lifetime values of appropriate excited levels. However, because of difficulties in measuring complete sets of transitions involved in the decay of a given level, and because of scarcity of reliable data for many levels, a procedure is often applied allowing the normalization to be expanded over transitions originating from other excited levels [5-7]. In such cases the knowledge of the plasma temperature and electron density is of great significance.

The knowledge of the electron density is crucial, e.g., for justification of the statistical population among excited levels (LTE or PLTE conditions $[8,9]$ ), while the knowledge of the temperature allows one, by applying the Boltzmann law, to interrelate the population of numerous excited levels to those used as references for normalization. In this manner the number of relative $A_{k i}$-values normalized to an absolute scale may be substantially increased [5-7]. 
The electron density is often obtained by taking advantage of the fact that usually the applied gases contain impurities of hydrogen, enabling the $\mathrm{H}_{\beta}$ line to be measured. The broadening of this line, which is mainly caused by the linear Stark effect, allows one to evaluate the electron density of the plasma $[10,11]$.

Numerous experimental studies have shown that helium arc plasmas at atmospheric pressure exhibit large departures from LTE model, see for example Ref. [12]. This is mainly caused by the small cross section of helium atoms, for both elastic and inelastic collisions [13]. Thus, the determination of the arc temperature based on measured intensities of helium spectral lines, may lead to temperatures which are far from those characterizing the population of excited levels of the admixture element. Moreover, because of the so-called demixing effect [14], and the long diffusion lengths for excited helium atoms [13], the temperatures based on HeI emission obtained for a selected plasma volume, might not reflect the electron energy distribution, which determines the population among excited levels of the studied element in this particular volume.

Because of the large energy gap in the helium atomic system, and the short lifetime of the level $1 s 2 p^{1} P_{1}^{\circ}$ (strong radiative transition to the level $1 s^{2}{ }^{1} S_{0}$ ), the ground and the metastable levels of HeI are overpopulated relative to levels with higher, $n \geq 3$, principal quantum numbers. Usually, this effect is taken into account by introducing a correction factor $\left(1 / b_{p}\right)$ in the Boltzmann law. In the case when the ground level is involved, this modified Boltzmann relation has the following form:

$$
N_{k} / N_{1}=\left(1 / b_{1}\right)\left(g_{k} / g_{1}\right) \exp \left(-E_{k} / k T\right),
$$

where $b_{1}$ is the measure of the overpopulation of the ground level, the other symbols $N, g, E$, and $k T$ have their usual meaning, and the subscripts " $k$ " and "1" indicate the excited and ground level, respectively.

The overpopulation factor " $b_{p}$ " is a complex function of the electron density, the temperature and optical thickness of the plasma at wavelengths corresponding to resonance transitions (see for example [15-17]). Therefore, the determination of helium densities in the ground and metastable states, from emission measurements in $\mathrm{HeI}$ and application of a pertinent "corrected" Boltzmann law, may lead to real uncertainties in the evaluated densities. Thus, the relation which is often applied within the LTE or PLTE model

$$
p=N_{\mathrm{e}} k T_{\mathrm{e}}+\Sigma_{\mathrm{a}} N_{\mathrm{a}} k T_{\mathrm{a}}+\Sigma_{\mathrm{i}} N_{\mathrm{i}} k T_{\mathrm{i}},
$$

where $p$ is the total plasma pressure, $N_{\mathrm{e}}, N_{\mathrm{a}}$ and $N_{\mathrm{i}}$ are the electron, atom and ion densities, may cause significant errors in the resulting plasma parameters (particle densities and temperature).

Because of the large differences between masses and mobilities of electrons and ions, the ion temperature $\left(T_{\mathrm{i}}=T_{\mathrm{a}}\right)$ of arc plasmas at atmospheric pressure is expected to be always somewhat lower than the electron temperature $T_{\mathbf{e}}$ [18]. Experiments with "extinguishing" arcs, where line intensity courses have been studied, confirm this outcome. From measurements of initial enhancement and subsequent decay of line intensities, the gas temperature before turning off the electric field supply, can easily be deduced (see for example $[19,20]$ ). 
Summing up all above comments - for studies of spectral properties of admixtures in helium plasmas it is prudent to determine the temperature from the spectrum of the admixture itself. Evaluations of plasma parameters which do not rely on questionable assumptions as LTE or PLTE, are of great value for reliable interpretation of the measured spectra and determination of atomic structure data.

In the following study we determine the main plasma parameters: the electron density and temperature only on the basis of line shape measurements, where the concept of LTE or PLTE is not as crucial as in the case of interpretation of speetral line intensities.

\section{Experiment}

For the purpose of this study we have prepared a cylinder with a gas mixture of $95 \% \mathrm{He}$ and $5 \%$ of Ar (by volume). The applied helium and argon gases contain traces of hydrogen, allowing the spectral intensity distribution of the Balmer $\beta$ line to be measured. The plasma was produced at atmospheric pressure in a wall-stabilized arc, with a channel diameter of $4 \mathrm{~mm}$ and a length of about $8 \mathrm{~cm}$. Two experiments were performed at currents of 30 and 60 amperes. For the analysis of the plasma emission in end-on direction two spectroscopic arrangements were set up.

(1) A grating spectrograph PGS2, equipped with an exit slit and a photomultiplier was used to perform spectral scans by turning the spectrograph's grating. The optical system focusing the light onto the entrance slit allows us to select the radiation originating from the plasma volume close adhering to the arc axis (spatial resolution of about $0.2 \mathrm{~mm}$ ). Spectra were taken in two wavelength intervals: (a) corresponding to the $\mathrm{H}_{\beta}$ radiation (4800-4920 $\AA$ ) and (b) where appropriate argon lines appear (4340-4355 $\AA$ ). Both intervals were scanned at least several times at steady state conditions for the running arc. The stability of the arc was checked by measuring the radiation emerging in the direction opposite to the main detection system. For this purpose a small monochromator was used. From spectral scans (b) the intensity ratios of two transitions close spaced in wavelength, ArII $4348 \AA$ /ArI $4345 \AA$, were measured.

At the plasma conditions and the resolution of our spectroscopic instrumentation, these two lines are well isolated and thus the intensity ratio could easily be evaluaied. The directly measured ratios require only a small correction resulting from the very weak wavelength dependence of the response of our detection system. This correction was carried out by calibrating the measured signal against that of a tungsten strip reference standard source. The signal from the same standard source was used for converting the directly measured $\mathrm{H}_{\beta}$ line shape into the line profile.

(2) The second spectroscopic setup consisted of a Fabry-Perot (FP) interferometer, a Jamin interferometer and again the PGS2 spectrograph with a small aperture and the photomultiplier behind the exit slit. Here the spectrograph facilitated to maintain the same spatial resolution and served as a narrow band filter. By setting the spectrograph's grating the required line was selected, while the spectral scanning was achieved in this small wavelength interval by changing very 
slowly the air pressure in the FP chamber. The calibration of the changes of the optical path in the FP, was accomplished with the $1 \mathrm{~m}$ Jamin interferometer by connecting one of its arms directly to the FP chamber. As a standard wavelength source a $\mathrm{He}-\mathrm{Ne}$ laser was applied. With this setup we measured the shapes of two ArII lines at 4348 and $4806 \AA$, for which the instrumental full widths at half maximum (FWHM) were 0.53 and $0.65 \mathrm{pm}$, respectively. The measured shapes are shown in Fig. 1 for both arc currents 30 and $60 \mathrm{~A}$. Details about the experimental setup, including the scheme of the optical system are described in Ref. [21].
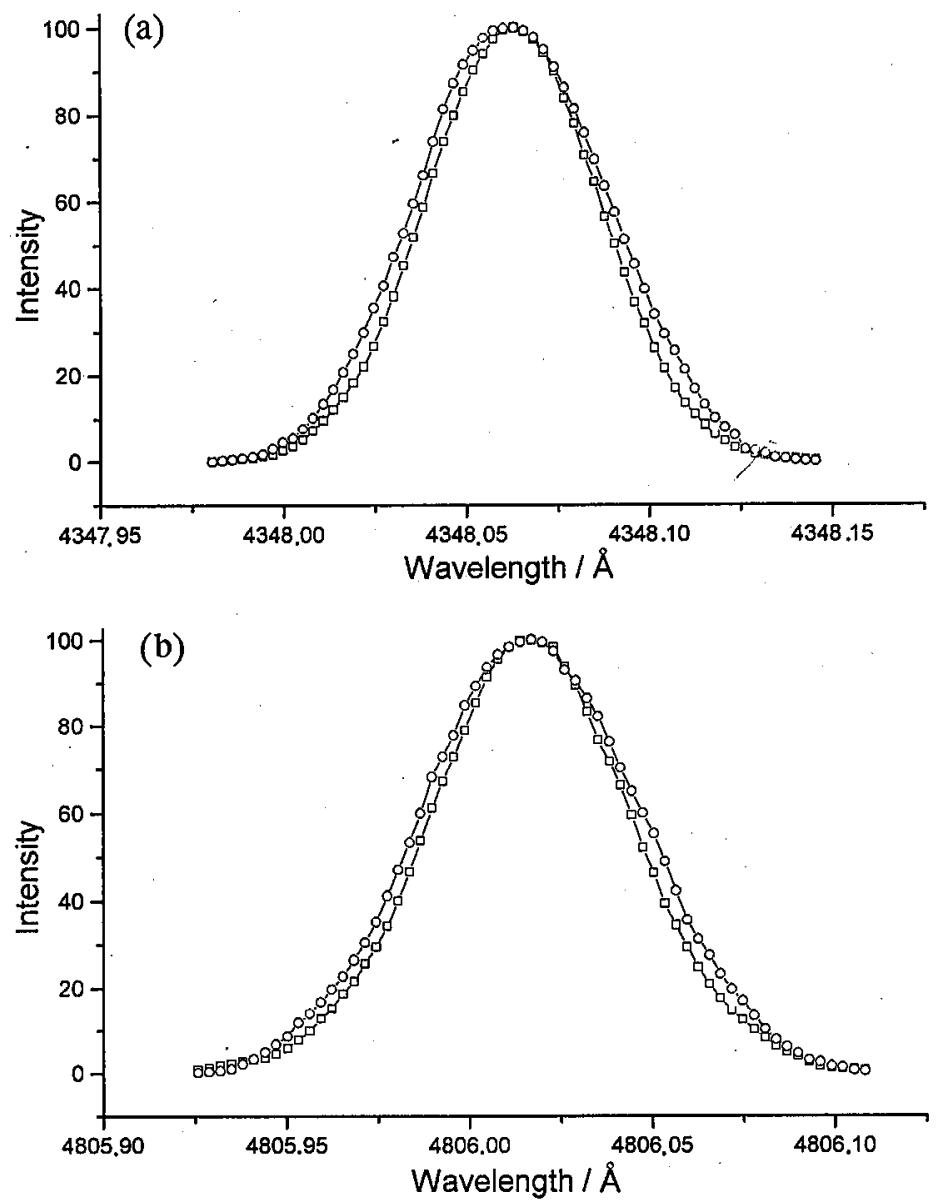

Fig. 1. Intensity distributions of ArII lines at $4348.063 \AA$ (a) and $4806.017 \AA$ (b) measured by applying the Fabry-Perot interferometer at two arc currents: $30 \mathrm{~A}$ (squares) and $60 \mathrm{~A}$ (circles). The wavelength steps correspond to the intervals between two subsequent maxima of the "calibration" signal from the Jamin interferometer $(0.00275$ and $0.00304 \AA$ for the lines 4348 and 4806 , respectively). 


\section{Evaluation of electron densities and temperatures of the plasma}

For the purpose of electron density determination we have measured the intensity distribution within the $\mathrm{H}_{\beta}$ line. After corrections of the wavelength dependence of the response of our detection system and after subtraction of the estimated Doppler contribution, we obtained the required Stark FWHM of the line for both experiments. The FWHM of the Doppler components did not exceed $8 \%$ of the corresponding Stark widths, for both experiments. According to two commonly applied theories [10;11], the FWHM of the $\mathrm{H}_{\beta}$ line mainly depends on the electron density and only insignificantly on the electron temperature. In Table I we quote the results obtained applying the theoretical broadening data taken from Refs. [10] and [11] and estimating the temperatures to be about 12000 and $14500 \mathrm{~K}$, for the experiments at currents 30 and $60 \mathrm{~A}$, respectively.

\section{TABLE I}

Electron densities of the plasma on the arc axis determined from the FWHM of the $\mathrm{H}_{\beta}$ line.

\begin{tabular}{c|c|c|c}
\hline \hline \multirow{2}{*}{ Experiment } & \multicolumn{3}{|c}{$\begin{array}{l}\text { Electron density (in } 10^{15} \mathrm{~cm}^{-3} \text { ) } \\
\text { based on theoretical data of }\end{array}$} \\
\cline { 2 - 4 } & Ref. [10] & Ref. [11] & Refs. [29, 30] \\
\hline 30 & $4.3 \pm 0.3$ & $4.0 \pm 0.3$ & $3.55 \pm 0.25$ \\
60 & $8.8 \pm 0.6$ & $8.2 \pm 0.6$ & $7.37 \pm 0.51$
\end{tabular}

However, in both theoretical approaches the contribution to the line width arising from ion dynamic effects is not included - the ions are considered as static perturbers. At expected plasma parameters of our experiments $\left(N_{\mathrm{e}}<10^{16} \mathrm{~cm}^{-3}\right.$, $T>10^{4} \mathrm{~K}$ ) these ion dynamic effects are not negligible for the formation of the $\mathrm{H}_{\beta}$ line profile, see for example Refs. [22, 23].

On the other hand the FWHM of ArII lines, are expected to be a result (convolution) of Doppler and Stark broadening (except for the very small instrumental broadening). Other broadening mechanisms do not affect the ArII line profiles at our plasma conditions.

In contrast to the case of $\mathrm{H}_{\beta}$, the contribution from Stark effect is expected to be small compared to the Doppler effect. (For all other lines appearing in the spectrum (HeI, ArI and H) the broadening caused by the Stark effect should be at least one order of magnitude larger than for any line of the ArII spectrum [24-26].)

Therefore, in order to determine the electron density and the temperature of the plasma we proceeded in an iterative way.

(1) For evaluation of the Stark component of our ArII lines we have applied the half-widths obtained by Helbig and Nick [27] and the electron density values listed in column 3 of Table I (based on Griem's broadening parameters). By comparing the directly measured line profile with the convolution of the evaluated Stark profile, the instrumental profile (Airy function), and a Gauss profile, the last contribution (the Doppler width) was determined. From these Doppler widths the plasma temperatures (gas or ion temperatures) have been calculated from the 
relation [28]

$$
T=1.95 \times 10^{12} M\left(\Delta \lambda_{1 / 2} / \lambda\right)^{2},
$$

where $T$ is given in kelvins, $M$ is the mass of the emitter (in atomic units), $\Delta \lambda_{1 / 2}$ and $\lambda$ are the FWHM and the wavelength of the selected spectral line, respectively.

(2) Applying the temperatures evaluated in the first step of our procedure, we determined again the Stark component of the $\mathrm{H}_{\beta}$ line from the measured spectrum. Then results of $\mathrm{H}_{\beta}$ line broadening calculations, which include ion dynamic effects, were used in our next step of the analysis. These calculations were performed for the $N_{\mathrm{e}}$ and $T$ range of our plasma conditions by Olchawa [29], according to the simulation technique approach described by Halenka and Olchawa [30]. In this way we obtained new and, as expected, slightly lower electron densities for both experiments. After indispensable corrections to ArII profiles, arising from Stark broadening (slightly narrower Lorentz profiles), new Doppler components were evaluated by fitting the new convolutions again to the directly measured profiles. These new Doppler widths were used to calculate new temperatures from Eq. (4).

As an example of our evaluation procedure in Fig. 2 the results for the $4348 \AA$ at the arc current of $30 \mathrm{~A}$ are shown (squares) together with the final fit (solid line). To compare the two main components, the Doppler profile (dashed line) and the Stark profile (dotted line) are also shown.

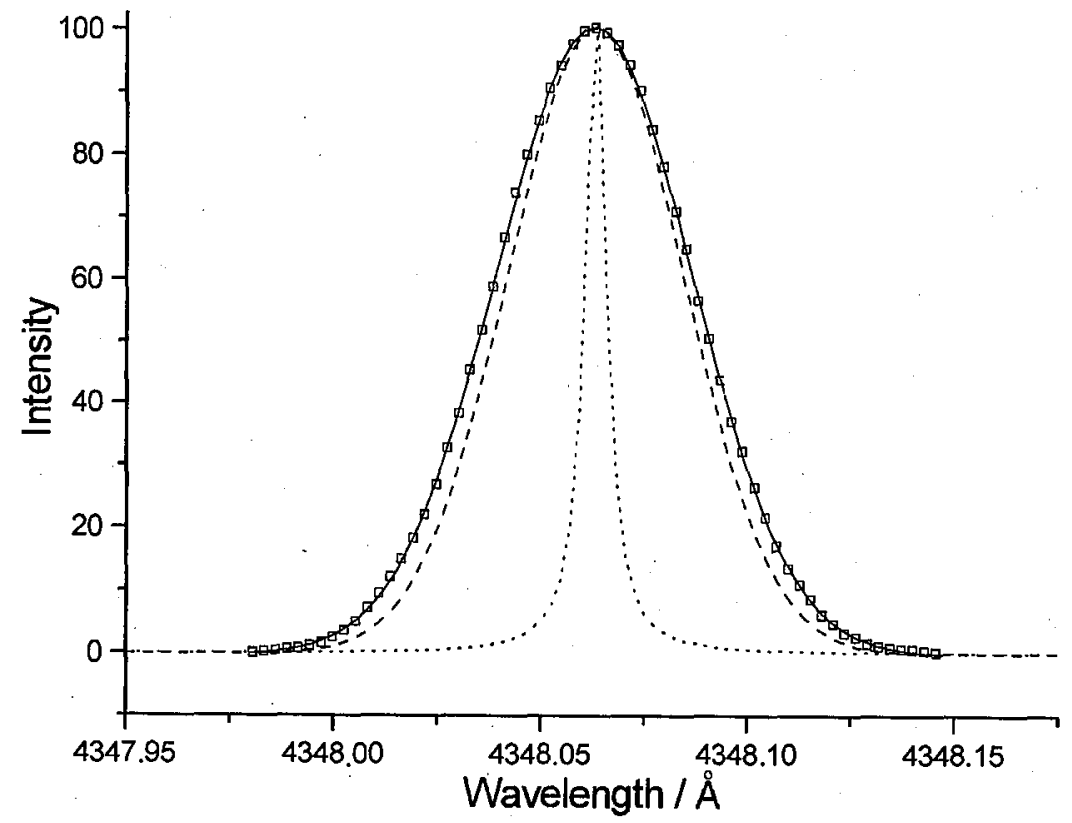

Fig. 2. Comparison of the measured profile of the $4348 \AA$ spectral line at 30 A (squares), with the final fit (solid line), the Doppler component (dashed line) and the Stark component (dotted line). All profiles are normalized to the maximum value of 100 at the line center. 
(3) It turns out that subsequent steps were not necessary to carry out, since the new temperatures taken for calculations of new FWHM of the $\mathrm{H}_{\beta}$ line, lead to $N_{e}$ values which were only insignificantly different from those obtained in the preceding step. In this way the final electron densities from measured $\mathrm{H}_{\beta}$ widths and theoretical results based on the simulation technique approach have been determined. The resulting $N_{\mathrm{e}}$ values are listed in the last column of Table I. The uncertainties quoted in Table I result from the accuracy of the determination of the FWHM of the $\mathrm{H}_{\beta}$ line, including the possible error introduced by the "subtraction" of the corresponding Doppler contribution but do not comprise the uncertainties of the applied theoretical input data. As can be seen from Eq. (4), the Doppler width of a line show only a weak dependence on temperature $\left(\Delta \lambda_{1 / 2}^{\text {Doppler }} \sim T^{1 / 2}\right)$. Therefore, a relative small variation of $\Delta \lambda_{1 / 2}$ causes significantly larger changes in $T$. Thus, in order to apply this method for $T$-determination, high accuracy measurements and evaluations are required. Particularly, the procedure of evaluation of the Doppler profile described above, may lead to real uncertainties if the Stark line width becomes comparable to the Doppler width.

\section{TABLE II}

Temperatures of the plasma on the arc axis obtained from Doppler broadening of ArII lines and from intensity ratios of two lines originating from ionized and neutral argon.

\begin{tabular}{c|c|c|c}
\hline \hline \multirow{3}{*}{ Experiment } & \multicolumn{4}{|c}{ Temperature in kelvins, obtained from } \\
\cline { 2 - 4 } I/A & the Doppler broadening of & the line intensity ratio \\
\cline { 2 - 4 } & ArII $4806 \AA$ & ArII $4348 \AA$ & ArII $4348 \AA /$ ArI $4345 \AA$ \\
\hline 30 & $12200 \pm 1200$ & $11700 \pm 1200$ & 14000 \\
60 & $14800 \pm 1600$ & $14500 \pm 1600$ & 16850
\end{tabular}

In Table II we compare the temperatures determined from Eq. (4) for our two experiments. The results obtained from broadening of both ArII lines are listed. The quoted errors result from the reproducibility of the measured spectra and from uncertainties of determination of the Doppler component by fitting the convolution of all significant contributions to the measured profile.

The errors $\Delta T$ significantly prevail the differences between the results obtained from the two ArII lines in both experiments. Such good agreement seems to indicate that this method is reliable for $T$-determination.

In order to compare our gas (ion) temperatures resulting from Doppler broadening of ArII lines with the electron temperature, we applied the measured intensity ratio of two argon lines in two subsequent ionization stages: the atomic line at $4345 \AA$ and the ionic line at $4348 \AA$. The ionic line originates from the $4 p^{4} D_{7 / 2}^{\circ}$ level with an excitation energy of $19.49 \mathrm{eV}$, while the atomic line from the level $5 p^{\prime}[3 / 2]_{1}$ with the $E_{k}$-value of $14.68 \mathrm{eV}$ [31]. For evaluation of these temperatures we have applied the electron densities quoted in the last column of Table I, and assumed that the ionization equilibrium is given by the Saha equation. The last 
assumption is obviously not entirely justified at our plasma conditions. One has rather to expect an equilibrium which results from collisional-radiative models applicable at lower $N_{\mathrm{e}}$ values. However, such models available in literature cannot be directly applied to our plasma, because they are valid for pure argon plasmas only - see for example Refs. [32-35].

Therefore, the temperatures evaluated by applying the Saha equation have to be regarded as the ones determining the lower limit for the electron temperature. In the last column of Table II we quote these temperatures. The corresponding transition probabilities for these lines have been taken from Refs. [36] and [37] for the atomic and ionic line, respectively.

\section{Conclusions}

Our measurements show that the electron temperatures indeed systematically prevail the gas temperatures by about $16 \%$, which is significantly more than expected from fundamental considerations of the plasma heating by the external electric field [18]. Moreover, the interpretation of the line intensity ratios within an appropriate collisional-radiative model, as mentioned earlier, would result even in higher temperatures.

However, there is a peculiarity which has to be considered when interpreting the results of our helium-argon plasma. The mean kinetic energy of the particles amounts about $1 \mathrm{eV}$ and $1.2 \mathrm{eV}$ for our two experiments, respectively. Many. prominent ArII lines in the visible range of the spectrum (among them our line at $4348 \AA$ ) have excitation energies about $19.2-21.5 \mathrm{eV}$. Thus one can expect that these $\mathrm{Ar}^{+}$levels may be very efficiently populated by inelastic collisions of $\mathrm{Ar}^{+}$ ions in the ground state with He-atoms in the $1 s 2 s{ }^{3} S_{1}$ and $1 s 2 s{ }^{1} S_{0}$ metastable states with their excitation energies 19.8 and $20.6 \mathrm{eV}$, respectively. Such collisions may cause a significant overpopulation of these $\mathrm{Ar}^{+}$levels with respect to the population expected from the Saha equilibrium as well as from the corrected Boltzmann distribution and thus lead to higher $T_{\mathrm{e}}$ values. Because of all these restrictions concerning the determination of the electron temperature, we do not quote any uncertainties for $T_{\mathrm{e}}$ in Table II.

The method of evaluation of the main plasma parameters applied in this work to an argon admixture in a helium arc plasma reveals some limitations and some advantages.

The basic condition for reliable gas temperature determination is to select and apply pertinent ionic lines with well-known (accurate) and small Stark widths (FWHM $\leq 0.3 \mathrm{pm}$ at $N_{\mathrm{e}}=10^{15} \mathrm{~cm}^{-3}$ ). The method may be successfully applied for helium plasmas only at electron densities below $10^{16} \mathrm{~cm}^{-3}$. The results of our work show also that electron densities determined on the basis of $\mathrm{H}_{\beta}$ broadening data, resulting from theoretical approach which include ion dynamic effects, are about $20 \%$ and $11 \%$ smaller than those obtained from "statical" data of Vidal, Cooper and Smith [10] and Griem [11], respectively.

To the main advantages of the procedure we have applied, we are reckoning: (i) the method does not require to consider a specific equilibrium model (LTE, PLTE) determining the population among excited levels of atoms and ions, and (ii) light intensity measurements in relative units are sufficient for determination 
of plasma parameters. In the case when high accuracy data are not requested, even a calibration of the wavelength dependence of the response of the detection system is not necessary, only a check of the linearity of the response at a given wavelength is required.

\section{Acknowledgments}

The authors would like to thank J. Halenka and W. Olchawa for discussions of ion dynamic effects and providing results of calculations of $\mathrm{H}_{\beta}$ line widths for several $N_{\mathrm{e}}$ and $T$ values.

\section{References}

[1] K. Dzierżęga, S. Łabuz, K. Musioł, J. Quant. Spectrosc. Radiat. Transfer 51, 567 (1994).

[2] J. Musielok, J.M. Bridges, S. Djurović, W.L. Wiese, Phys. Rev. A 53, 3122 (1996).

[3] J. Musielok, G. Veres, W.L. Wiese, J. Quant. Spectrosc. Radiat. Transfer 57, 395 (1997).

[4] J. Musielok, W.L. Wiese, S. Glenzer, H.-J. Kunze, J. Quant. Spectrosc. Radiat. Transfer 61, 215 (1999).

[5] J. Musielok, W.L. Wiese, G. Veres, Phys. Rev. A 31, 3588 (1995).

[6] J.A. Aparicio, M.A. Gigosos, S. Mar, J. Phys. B 30, 3141 (1997).

[7] J. Musielok, E. Pawelec, U. Griesmann, W.L. Wiese, Phys. Rev. A, submitted.

[8] H.R. Griem, Principles of Plasma Spectroscopy, Cambridge University Press, Cambridge 1997.

[9] H.W. Drawin, Z. Naturforsch. A 19, 1451 (1974).

[10] C.R. Vidal, J. Cooper, E.W. Smith, Astrophys. J., Suppl. Ser. 25, 37 (1973).

[11] H.R. Griem, Spectral Line Broadening by Plasmas, Academic Press, New York 1974.

[12] J. Uhlenbusch, E. Fischer, J. Hackmann, Z. Phys. 239, 120 (1970).

[13] H.R. Griem, Plasma Spectroscopy, Mc Graw-Hill, New York 1964.

[14] W. Frie, H. Maecker, Z. Phys. 168, 208 (1962).

[15] H.W. Drawin, F. Emard, Z. Naturforsch. A 28, 1239 (1973).

[16] T. Fujimoto, J. Quant. Spectrosc. Radiat. Transfer 21, 439 (1979).

[17] H.C. Srivastava, P.K. Ghosh, J. Quant. Spectrosc. Radiat. Transfer 25, 59 (1981).

[18] W.L. Wiese, Electric Arcs, in series Methods of Experimental Physics, Vol. 7B, Academic Press, New York 1968.

[19] H. Kaufroni, Physica C 98, 100 (1979).

[20] K.-P. Nick, J. Richter, V. Helbig, J. Quant. Spectrosc. Radiat. Transfer 32, 1 (1984).

[21] A. Goly, G. Łopatka, T. Wujec, J. Quant. Spectrosc. Radiat. Transfer 47, 353 (1992).

[22] C. Stehle, Astron. Astrophys. Suppl. Ser. 104, 509 (1994).

[23] M.A. Gigosos, V. Cardeñoso, J. Phys. B 29, 4795 (1996).

[24] N. Konjević, W.L. Wiese, J. Phys. Chem. Ref. Data 5, 259 (1976). 
[25] N. Konjević, M.S. Dimitrijević, W.L. Wiese, J. Phys. Chem. Ref. Data 13, 619 (1984).

[26] N. Konjević, W.L. Wiese, J. Phys. Chem. Ref. Data 19, 1307 (1990).

[27] K.-P. Nick, V. Helbig, Phys. Scr. 33, 55 (1986).

[28] W.C. Martin, W.L. Wiese, Atomic Spectroscopy, in Atomic, Molecular and Optical Physics Handbook, Ed. G. Drake, Am. Inst. of Physics, Woodbury, New York 1996.

[29] W. Olchawa, unpublished.

[30] J. Halenka, W. Olchawa, J. Quant. Spectrosc. Radiat.' Transfer 56, 17 (1996).

[31] A.R. Striganov, G.S. Svientitskii, Tablicy Spiektralnych Linii, Atomizdat, Moskwa 1966 (in Russian).

[32] K. Katsonis, Ph.D. Thesis, Univ. de Paris-Sud, Centre d'Orsay 1976.

[33] C.G. Braun, J.A. Kunc, Phys. Fluids 31, 671 (1988).

[34] J. Vlcek, V. Pelikan, J. Phys: D, Appl. Phys. 22, 632 (1989).

[35] D.A. Benoy, J.A.M. van der Mullen, B. van der Sijde, D.C. Schram, J. Quant. Spectrosc. Radiat. Transfer 46, 195 (1991).

[36] W.L. Wiese, J.W. Brault, K. Danzmann, V. Helbig, M. Kock, Phys. Rev. A 39, 2461 (1989).

[37] V. Vujnović, W.L. Wiese, J. Phys. Chem. Ref. Data 21, 919 (1992). 